\title{
ANÁLISE DE SENSIBILIDADE DOS PARÂMETROS DO MODELO SWAT E SIMULAÇÃO DOS PROCESSOS HIDROSSEDIMENTOLÓGICOS EM UMA BACIA NO AGRESTE NORDESTINO ${ }^{(1)}$
}

\author{
Ricardo de Aragão ${ }^{(2)}$, Marcus Aurélio Soares Cruz ${ }^{(3)}$, Julio Roberto Araujo de Amorim ${ }^{(3)}$, \\ Luciana Coêlho Mendonça ${ }^{(2)}$, Eduardo Eneas de Figueiredo ${ }^{(4)}$ \& Vajapeyam S. Srinivasan $^{(5)}$
}

RESUMO

\begin{abstract}
O processo erosivo constitui-se na principal causa de degradação dos solos e é acelerado por intervenções humanas nas bacias hidrográficas, resultando em prejuízos ao setor agrícola e ao meio ambiente. Para a previsão dos impactos de ações antrópicas ou de mudanças climáticas sobre os processos hidrossedimentológicos, os modelos distribuídos e de base física têm sido bastante eficazes. Neste estudo, aplicou-se o modelo SWAT à sub-bacia do Rio Japaratuba Mirim, em Sergipe, nas seções de medição das estações Fazenda Pão de Açúcar (PA) e Fazenda Cajueiro (CJ), com áreas de contribuição respectivamente de 137,3 e 277,8 km$^{2}$, visando à parametrização das duas bacias aninhadas por meio dos processos de calibração e de validação. Foram realizadas simulações do escoamento na bacia menor (PA), a partir dos parâmetros calibrados na bacia maior (CJ), e, reciprocamente, do escoamento na bacia maior (CJ). Em seguida, foram também realizadas simulações do escoamento e da produção de sedimentos para o período de dados de vazão disponíveis (1985 a 2000), para a seção CJ. Os resultados evidenciaram que o modelo calibrado simulou bem o escoamento superficial e conseguiu prever, de forma coerente, a produção de sedimentos com base nos 12 parâmetros mais sensíveis do modelo. A transposição dos parâmetros da bacia maior para a menor e, vice-versa,
\end{abstract}

(1) Recebido para publicação em 3 de outubro de 2012 e aprovado em 17 de abril de 2013.

${ }^{(2)}$ Professor da Universidade Federal de Sergipe - UFS. Av. Marechal Rondon, s/n, Jardim Rosa Elze. CEP $49100-000$ São Cristóvão (SE). E-mail: ricardoaragao@yahoo.com; lumendon@uol.com.br

(3) Pesquisador da Embrapa Tabuleiros Costeiros. Av. Beira Mar, 3250, Jardins. CEP 49025-040 Aracaju (SE). E-mail: marcus.cruz@embrapa.br; julio.amorim@embrapa.br

(4) Professor da Universidade Federal de Campina Grande, UFCG. Caixa Postal 505. CEP 58.100-970 Campina Grande (PB). E-mail: eneasef@yahoo.com

(5) Professor Visitante Nacional Sênior, Centro Acadêmico do Agreste, Universidade Federal de Pernambuco - UFPE. Rod. BR 104, km 59, s/n, Sítio Juriti, Zona Rural. CEP 55002-970 Caruaru (PE). E-mail: vajapeyam@yahoo.com 


\begin{abstract}
resultou em índices de Eficiência de Nash-Sutcliffe (NSE) e de Tendência Percentual (PBIAS), considerados satisfatórios para o primeiro caso e insatisfatório para o segundo.
\end{abstract}

Termos de indexação: erosão hídrica, produção de sedimentos, modelagem hidrológica.

\title{
SUMMARY: SENSITIVITY ANALYSIS OF THE PARAMETERS OF THE SWAT MODELAND SIMULATIONOF THE HYDROSEDIMENTOLOGICAL PROCESSES IN A WATERSHED IN THE NORTHEASTERN REGION OF BRAZIL
}

\begin{abstract}
Erosion has been recognized as the main cause of soil degradation and is accelerated by human intervention in watersheds, causing losses to the agricultural sector and damaging the environment. To estimate the impacts caused by land use or climate changes on hydrosedimentological processes, physically-based distributed models have been shown to be quite effective. In this study, the SWAT model was calibrated and validated for two subwatersheds of the Japaratuba Mirim river watershed, one located upstream of the Fazenda Pão de Açúcar - PA (137.3 km²), and another located upstream of Fazenda Cajueiro - CJ $\left(277.8 \mathrm{~km}^{2}\right)$ in the state of Sergipe, to simulate runoff and soil erosion. To test the sensitivity of the calibrated parameters, the runoff was also simulated by a cross application of the 12 most sensitive parameters in the two watersheds, from 1985 to 2000. The results showed that the model was able to simulate the runoff and forecast, in a consistent way, the sediment yield. However, while the cross application of the parameters from the bigger (CJ) to the smaller watershed (PA) resulted in satisfactory Nash-Sutcliffe efficiency (NSE) and percent bias (PBIAS), the opposite was not true.
\end{abstract}

Index terms: water erosion, sediment yield, hydrologic modeling.

\section{INTRODUÇÃO}

A erosão do solo resulta da ação conjunta dos processos de desagregação por impacto, transporte e deposição de partículas sólidas, provocados pelas forças erosivas das gotas de chuva e do fluxo superficial. Quando acelerada, principalmente pelo desmatamento, a erosão causa impactos ambientais, econômicos e sociais (Bertol et al., 2007; Pugliesi et al., 2011). O desmatamento também contribui para o aumento do escoamento superficial e da produção de sedimentos, com perda de nutrientes e redução da capacidade produtiva dos solos (Araújo \& Knight, 2005), além de acarretar o assoreamento de reservatórios e rios (Bertoni \& Lombardi Neto, 2005).

Sendo assim, para o manejo integrado das bacias hidrográficas, faz-se necessário o conhecimento sobre o escoamento superficial gerado e a consequente carga de sedimento, em razão da erosão do solo pelos eventos naturais da chuva. Nesse sentido, diversos são os métodos para quantificação do volume escoado e a descarga de sedimentos até uma dada seção da bacia (Lane et al., 1997; Araújo \& Knight, 2005). Relativamente ao escoamento, a medição da descarga de sedimento é mais complexa em grandes bacias (Singh \& Woolhiser, 2002). Nessas, na maioria das vezes, apenas uma parcela da carga de sedimento em suspensão é possível ser medida, sendo, portanto, inferior à erosão total por causa do desprendimento pela chuva e escoamento (Dedkov, 2004; Araújo \& Knight, 2005).

Considerando a dificuldade em se quantificar a produção de sedimentos em grandes bacias, bem como o efeito da escala da bacia sobre os processos hidrossedimentológicos, sua estimativa tem sido realizada por meio de modelos de simulação, sendo, em geral, relacionados com as características do solo (tamanho das partículas de sedimento, densidade etc.), com os fatores climáticos (chuva e evaporação) e os fisiográficos (área da bacia, relevo e cobertura vegetal). Ademais, a relação depende da distribuição espacial desses fatores, do uso e da cobertura do solo na área da bacia, bem como da tendência de ocorrência de chuvas intensas e sua variabilidade espacial (Walling \& Webb, 1996; Dedkov, 2004; Birkinshaw \& Bathurst, 2006; Lima et al., 2008).

A modelagem consiste basicamente na parametrização de um modelo voltado para a simulação de processos. A simulação hidrossedimentológica distribuída e de base física (Beven, 2001; Singh \& Woolhiser, 2002; Merritt et al., 2003; Figueiredo, 2008) estima, entre outras informações, o volume de água e a carga de sedimentos para dada seção de um rio e, ou, reservatório, em razão de uma ou diversas chuvas naturais (Birkinshaw \& Bathurst, 2006). Quando os parâmetros têm aplicabilidade regional, também é 
possível a previsão de vazão em lugares onde não existem tais dados. Contudo, a disponibilidade de dados e a determinação adequada dos parâmetros de modelos distribuídos de base física têm sido as principais dificuldades na modelagem distribuída de processos naturais (Beven, 2001).

$\mathrm{O}$ acoplamento de sistemas de informações geográficas aos modelos hidrossedimentológicos (Melo, 2010) tem facilitado a utilização de modelos de base física; exemplo disso é o caso do modelo SWAT (Neitsch et al., 2005), acoplado a um SIG - ArcSWAT (Winchell et al., 2009), que tem produzido bons resultados no Brasil e em diversas partes do mundo (Jayakrishnan et al., 2005; Garbossa et al., 2011; Lelis et al., 2012; Strauch et al., 2012).

Este estudo teve como objetivo realizar a calibração do modelo SWAT, bem como a análise de sensibilidade dos parâmetros, com base em dados de duas bacias de contribuição para as estações Fazenda Pão de Açúcar (PA) e Fazenda Cajueiro (CJ), que são aninhadas e localizadas no Estado de Sergipe, visando simular o escoamento superficial e a erosão do solo, bem como verificar os resultados da transposição dos parâmetros calibrados de uma seção de medição para a outra, e vice-versa, entre as duas bacias.

\section{MATERIAL E MÉTODOS}

\section{Descrição da área de estudo}

A bacia hidrográfica do Rio Japaratuba $\left(10^{\circ} 13^{\prime} 00^{\prime \prime}\right.$ e $10^{\circ} 47^{\prime} 00^{\prime \prime}$ S e $36^{\circ} 48^{\prime} 00^{\prime \prime}$ e $37^{\circ} 19^{\prime} 00^{\prime \prime}$ O) é uma das seis grandes bacias que compõem o Estado de Sergipe, sendo a única totalmente estadual. O rio principal, que dá nome à bacia, tem extensão de $94 \mathrm{~km}$ (Figura 1). Com área de $1.687,67 \mathrm{~km}^{2}$, a bacia tem sido fortemente impactada nos últimos dois séculos

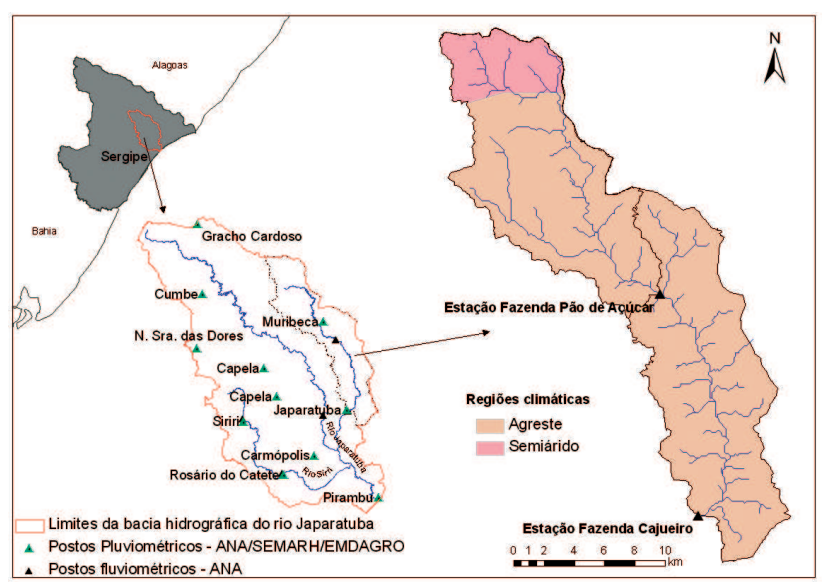

Figura 1. Bacia do Rio Japaratuba e a sub-bacia do Rio Japaratuba Mirim com as estações fluviométricas localizadas na Fazenda Cajueiro (CJ) e Fazenda Pão de Açúcar (PA). por atividades agropecuárias e de extrativismo mineral: petróleo e potássio (Aragão et al., 2011). Essa bacia contém a sub-bacia do Rio Japaratuba Mirim, principal contribuinte pela margem esquerda do Rio Japaratuba, com área de $335,4 \mathrm{~km}^{2}$ e equivalente a $20 \%$ da área total da bacia deste Rio.

Na sub-bacia do Rio Japaratuba Mirim, são identificadas duas regiões climáticas: agreste $\left(\mathrm{P}_{\text {média }} \sim 800 \mathrm{~mm}\right)$ e semiárido $\left(\mathrm{P}_{\text {média }} \sim 500 \mathrm{~mm}\right)$, que correspondem respectivamente a 89,4 e $10,6 \%$ de sua área total. O período de chuvas ocorre entre os meses de abril e agosto, sendo o intervalo de maio a junho o mais chuvoso. A temperatura média anual é de $25^{\circ} \mathrm{C}$ e a umidade relativa do ar, de aproximadamente $74 \%$ (SEMARH, 2004). Nessa sub-bacia, há duas estações fluviométricas monitoradas pela Agência Nacional de Águas (ANA): Fazenda Pão de Açúcar (PA) - código ANA 50042000 e Fazenda Cajueiro (CJ) - código ANA 50043000 (Figura 1), respectivamente com áreas das bacias de contribuição de 137,3 e $277,8 \mathrm{~km}^{2}$ e vazões médias anuais de 0,66 e $1,70 \mathrm{~m}^{3} \mathrm{~s}^{-1}$, respectivamente (ANA, 2012). Os dados de vazão coletados nas seções PA e CJ compreendem ao período de 1985 a 2000 (ANA, 2012). Os dados climatológicos disponíveis são provenientes da estação Fazenda Experimental Pirangi $\left(10^{\circ} 29^{\prime} \mathrm{S}\right.$ e $37^{\circ} 04^{\prime}$ O - código ANA 01037078), localizada no Município de Capela, coletados no período de 1983 a 2005 (ANA, 2012).

O substrato geológico da sub-bacia do Rio Japaratuba Mirim, conforme apresentado na figura 2a, é composto por Formações Barreiras (51,95\%), Coruripe $(7,56 \%)$, Depósitos aluvionares (6,9\%), Grupo Perucaba $(5,30 \%)$, arenitos médios, grosso e suíte intrusiva $(0,38 \%)$ e pelos membros Taquari e Maruim $(0,01 \%)$ e pelas rochas do embasamento cristalino, representadas pelo Grupo Macururé $(27,90 \%)$. No trecho de nascentes, há uma porção da Formação Barreiras, seguida do Grupo Macururé, ocupando $56 \%$ da área de contribuição para a estação PA. O trecho restante é composto por Formações Barreiras, Coruripe, depósitos aluvionares e Grupo Perucaba, até a área da estação CJ (Semarh, 2004). Na área da sub-bacia, os solos predominantes pertencem às seguintes classes (Embrapa, 2006): Argissolos Amarelos (134,9 km², 48,6\%), Argissolos VermelhoAmarelo (101,5 km², 36,5 \%), Neossolos Flúvicos $\left(24,1 \mathrm{~km}^{2}, 8,7 \%\right)$ e Neossolos Quartzarênicos $\left(17,3 \mathrm{~km}^{2}\right.$, $6,2 \%$ ) (Figura 2b). No quadro 1, encontram-se os valores médios de atributos físico-químicos (camada de 0-0,20 m) dos solos da sub-bacia, segundo Araújo Filho et al. (1999).

No período de condução do estudo (1985-2000), o uso e a cobertura do solo na sub-bacia (Figura 2f) eram, em porcentagem da área total, para a estação PA: cidades $(1,00 \%)$, floresta $(4,00 \%)$, mata secundária $(46,00 \%)$, pastagem $(32,00 \%)$ e área cultivada com cana-de-açúcar (17,00\%); e para a estação CJ: cidades $(0,03 \%)$, floresta $(4,97 \%)$, mata secundária $(25,00 \%)$, pastagem $(40,00 \%)$ e área cultivada com cana-de- 

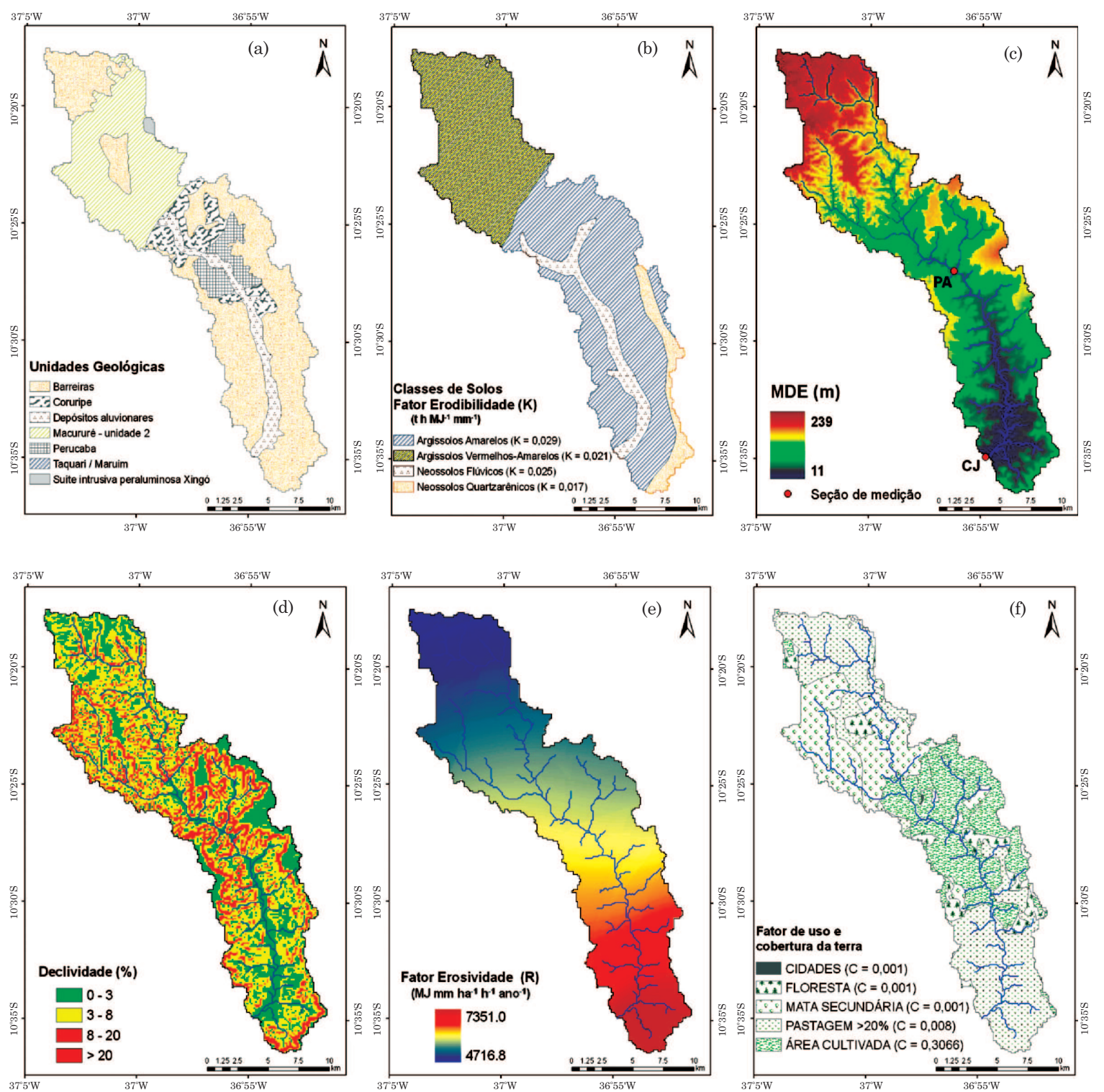

Figura 2. Mapas de unidades geológicas (a), classes de solos e fator erodibilidade - USLE (b), modelo digital de elevação (c), declividade (d), fator erosividade - USLE (e) e fator de uso e cobertura do solo - USLE (f), para a sub-bacia do Rio Japaratuba Mirim.

açúcar (30,00 \%) (Semarh, 2004). Na sub-bacia, não existem áreas significativas de exploração mineral.

\section{Modelagem hidrossedimentológica}

O SWAT (Soil and Water Assessment Tool) é um modelo hidrossedimentológico contínuo no tempo, de base física (as equações utilizadas pelo modelo são baseadas em leis da física) e distribuído (os parâmetros do modelo dependem do espaço e, ou, do tempo, permitindo com isso a representação da sua variabilidade espaço-temporal). O SWAT pode simular diversos processos em bacias hidrográficas, como escoamento superficial e subterrâneo, erosão e produção de sedimentos nos planos e nos canais, transportes de nutrientes e de pesticidas e contaminação dos recursos hídricos, na escala de bacia e no passo de tempo diário, sob o efeito de alterações no uso e na cobertura do solo e de impactos ambientais provocados pela ação antrópica (Baldissera, 2005; Neitsch et al., 2005; Meira Neto et al., 2011). Na abordagem do modelo SWAT, com base em tipo de solo, relevo, uso e cobertura do solo, a bacia hidrográfica é subdividida em sub-bacias e essas, por sua vez, em unidades de resposta hidrológica (HRU). Por ser distribuído, cada elemento, plano ou canal pode ter seu próprio conjunto de parâmetros que o caracteriza adequadamente. Desse modo, preservamse os parâmetros espacialmente distribuídos da bacia inteira e as características homogêneas dentro da bacia (Baldissera, 2005). 
Quadro 1. Valores médios de atributos físico-químicos, na camada de 0-0,20 m, dos solos da bacia do Rio Japaratuba Mirim

\begin{tabular}{|c|c|c|c|c|}
\hline \multirow[b]{2}{*}{ Atributo } & \multicolumn{4}{|c|}{ Classe de solo } \\
\hline & $\begin{array}{c}\text { Argissolo } \\
\text { Amarelo }\end{array}$ & $\begin{array}{c}\text { Argissolo } \\
\text { Vermelho-Amarelo }\end{array}$ & $\begin{array}{l}\text { Neossolo } \\
\text { Flúvico }\end{array}$ & $\begin{array}{c}\text { Neossolo } \\
\text { Quartzarênico }\end{array}$ \\
\hline Areia, $\mathrm{g} \mathrm{kg}^{-1}$ & 430 & 710 & 600 & 740 \\
\hline Silte, $\mathrm{g} \mathrm{kg}^{-1}$ & 330 & 160 & 240 & 100 \\
\hline Argila, g kg-1 & 240 & 130 & 160 & 160 \\
\hline Classe textural & Franca & Franco-arenosa & Franco-arenosa & Franco-arenosa \\
\hline $\mathrm{pH}\left(\mathrm{H}_{2} \mathrm{O}\right)$ & 5,80 & 4,40 & 4,90 & 4,80 \\
\hline Matéria orgânica, $\mathrm{g} \mathrm{kg}^{-1}$ & 29,10 & 17,20 & 19,80 & 13,30 \\
\hline $\mathrm{Ca}^{2+}, \mathrm{cmol}_{\mathrm{c}} \mathrm{dm}^{-3}$ & 5,07 & 0,67 & 0,53 & 0,27 \\
\hline $\mathrm{Mg}^{2+}, \mathrm{cmol}_{\mathrm{c}} \mathrm{dm}^{-3}$ & 1,20 & 0,67 & 0,33 & 0,27 \\
\hline $\mathrm{Al}^{3+}, \mathrm{cmol}_{\mathrm{c}} \mathrm{dm}^{-3}$ & 0,00 & 0,20 & 0,93 & 0,33 \\
\hline $\mathrm{H}+\mathrm{Al}, \mathrm{cmol}_{\mathrm{c}} \mathrm{dm}^{-3}$ & 3,33 & 2,33 & 5,93 & 3,33 \\
\hline Soma de bases (S) & 6,46 & 1,54 & 0,96 & 0,85 \\
\hline CTC, $\mathrm{cmol}_{\mathrm{c}} \mathrm{dm}^{-3}$ & 9,80 & 3,87 & 6,87 & 4,78 \\
\hline Saturação por bases (V), \% & 66,00 & 40,00 & 14,00 & 30,00 \\
\hline $\mathrm{Na}, \mathrm{mg} \mathrm{dm}^{-3}$ & 23,00 & 3,07 & 9,20 & 13,80 \\
\hline $\mathrm{K}, \mathrm{mg} \mathrm{dm}^{-3}$ & 21,47 & 44,47 & 12,27 & 59,80 \\
\hline $\mathrm{P}, \mathrm{mg} \mathrm{dm^{-3 }}$ & 2,00 & 0,67 & 0,67 & 4,13 \\
\hline PST, $\%$ & 1,02 & 0,34 & 0,58 & 1,26 \\
\hline
\end{tabular}

Fonte: Araújo Filho et al. (1999).

Para o SWAT, o transporte de sedimentos na rede de drenagem ocorre em razão de processos simultâneos de desagregação das partículas (cisalhamento hidráulico) e deposição no canal (Neitsch et al., 2005), cujo resultado é calculado por meio da equação simplificada sugerida por Bagnold (Williams, 1975), com base na definição de potência do fluxo. A perda de solo nos planos ou sub-bacias é calculada pela Equação Universal de Perda de Solo - USLE (Wischmeier et al., 1971; Neitsch et al., 2005), determinada pela relação $\mathrm{A}=\mathrm{R} \mathrm{KL} \mathrm{S} \mathrm{C} \mathrm{P,} \mathrm{em} \mathrm{que} \mathrm{A} \mathrm{=} \mathrm{perda} \mathrm{do} \mathrm{solo} \mathrm{por}$ unidade de área $\left(\mathrm{t} \mathrm{ha}^{-1}\right) ; \mathrm{R}=$ fator de erosividade da chuva (MJ mm ha-1 $\mathrm{h}^{-1}$ ano $^{-1}$ ); $\mathrm{K}=$ fator de erodibilidade do solo ( $\mathrm{t} \mathrm{h} \mathrm{MJ} \mathrm{Mm}^{-1} \mathrm{~mm}^{-1}$ ); $\mathrm{L}=$ fator de comprimento de declive; $\mathrm{S}=$ fator de grau de declive; $\mathrm{C}=$ fator de uso e manejo; e $\mathrm{P}=$ fator práticas conservacionistas. O acoplamento do SWAT a um SIG, o ArcSWAT, permite, entre outras coisas, a discretização da bacia em sub-bacias e a extração dos parâmetros do modelo, a partir de informações de classe de solo, declividade, uso e cobertura do solo (Winchell et al., 2009). O modelo utiliza, ainda, dados climatológicos diários, como precipitação, temperatura, umidade relativa do ar e velocidade do vento.

Em razão do grande número de equações representativas dos processos hidrossedimentológicos considerados no modelo SWAT, diversos parâmetros precisam ser calibrados ou determinados. Para identificar os parâmetros que exercem maior influência sobre os resultados gerados, são utilizados dois métodos de análise de sensibilidade (van
Griensven et al., 2006): Latin-Hypercube (LH) e Onefactor at a time (OAT). Por meio desses dois métodos e a partir de dados observados (vazão diária), a análise de sensibilidade é efetuada, mudando-se o valor de um parâmetro por vez a cada simulação executada e avaliando-se a resposta do modelo com relação aos dados medidos para fornecer uma classificação desses parâmetros, em razão do grau de sensibilidade à resposta desse modelo.

Essa análise, para cada uma das duas estações, foi realizada com utilização de dados de vazão média mensal do período 1985 a 1988, oriundos das estações CJ e PA. Como resultado, foram identificados 27 parâmetros como os mais influentes sobre os resultados gerados (Neitsch et al., 2005; van Griensven et al., 2006) e a cada um foi dado um número de ordem. O mesmo período foi também empregado para calibração automática dos parâmetros identificados.

Em virtude das incertezas quanto às condições iniciais de alguns parâmetros do modelo, como umidade inicial, crescimento da vegetação etc., fez-se necessário um período para ajustamento desses parâmetros, conhecido também como período de "aquecimento" do modelo. Para esse fim, utilizaramse os dados de 1985 (Neitsch et al., 2005), enquanto o período 1986 a 1988 foi usado para calibração dos parâmetros. Essa calibração foi efetuada por meio da rotina de autocalibração existente no próprio modelo, considerando-se as faixas de valores de cada parâmetro (Neitsch et al., 2005; van Griensven et al., 2006). A 
rotina emprega o método Parasol (Parameter Solutions method), que tem por base o algoritmo genético Shuffled Complex Evolution (SCE-UA) (Duan et al., 1994). A etapa de validação dos valores dos parâmetros para as áreas de contribuição de PA e CJ foi efetuada, considerando-se vazões do período de 1989 a 1990, por meio da análise dos hidrogramas observados e simulados e dos índices de Eficiência de Nash-Sutcliffe (NSE) e de Tendência Percentual (PBIAS), conforme quadro 2, para a avaliação da precisão dos dados gerados pelo modelo, em comparação àqueles observados (Moriasi et al., 2007; Meira Neto et al., 2011).

A possibilidade de se transpor ou utilizar os parâmetros calibrados para uma bacia em outra, na vizinhança e, ou, com condições semelhantes, foi considerada. Para esse fim, foram realizadas simulações na seção CJ da bacia de contribuição maior e na seção PA da bacia menor, com a utilização recíproca (transposição) dos parâmetros. O objetivo principal desses testes foi realizar a previsão de vazões em sub-bacias, para as quais não existem dados disponíveis, ou a previsão de vazões em uma bacia inteira, com disponibilidade de dados apenas em alguma(s) sub-bacia(s). Em seguida à calibração dos parâmetros relativos ao escoamento superficial, realizou-se a simulação hidrossedimentológica para a seção CJ (exutório da bacia maior) e para a PA (exutório da bacia menor aninhada à maior), para o período de 1986 a 2000, obtendo-se assim valores de vazão e carga de sedimentos nas duas seções das bacias. Salienta-se que, em razão da inexistência de dados observados sobre a produção de sedimentos nas bacias de contribuição, consideradas neste estudo, não foi possível a calibração dos parâmetros relativos ao processo erosivo.

A partir das informações relativas ao solo, ao uso e à ocupação, foram estimados alguns dos parâmetros que compõe a USLE (Neitsch et al., 2005), como: fator de erodibilidade do solo (fator K), por meio da aplicação da equação de Williams (1975) (Figura 2b); fator de erosividade da chuva (fator R), obtido a partir de
Aragão et al. (2011) (Figura 2e); e fator topográfico composto (L.S), determinado a partir do Modelo Digital de Elevação - SRTM (Miranda, 2005) (Figura 2d). Visto que não há informações disponíveis sobre o fator de uso e a cobertura do solo (fator C) para a sub-bacia em questão, foram utilizados os valores propostos por Weill \& Sparovek (2008) para cana-de-açúcar, pastagem, mata secundária e floresta (Figura 2f).

\section{RESULTADOS E DISCUSSÃO}

A área de contribuição para a estação PA foi dividida em 32 sub-bacias e a respectiva área para CJ foi dividida em 112 sub-bacias. Dos 27 parâmetros identificados na análise de sensibilidade, relacionados ao processo chuva-vazão, 12 apresentaram as maiores sensibilidades (Quadro 3) e foram empregados para calibração automática, de acordo com van Griensven et al. (2006) e Arnold et al. (1995). Não estão incluídos, no quadro 3, os parâmetros pouco influentes sobre os resultados (baixos número de ordem), 15 no total, sendo esses mantidos nos valores assumidos pelo modelo na ausência de calibração.

Para as duas bacias, os parâmetros Alpha_Bf, $C h \_K 2, C n 2$, Surlag, Sol_Z, Esco e Ch_N2 tiveram números de ordem iguais ou muito próximos, demonstrando a importância e influência sobre os resultados da simulação em ambas bacias. Tais parâmetros destacaram o escoamento subterrâneo nos processos simulados pelo modelo, demandando, portanto, dados relativos aos solos com elevado grau de confiabilidade. Em termos gerais, o modelo apresentou alto grau de sensibilidade aos parâmetros relacionados à água subterrânea, tendência essa também observada por Baltokoski et al. (2010) e Lelis et al. (2012).

Em se tratando da calibração, existe uma variação considerável entre os valores de alguns parâmetros para as estações CJ e PA, como é o caso do $C n 2$, Esco, Gw_Delay, GWrevap, Gwqmn e Sol Z, sendo os dois

Quadro 2. Índices de Eficiência de Nash-Sutcliffe (NSE) e de Tendência percentual (PBIAS) para avaliação do desempenho do modelo e da classificação da precisão dos dados de vazão e sedimentos, simulados em relação aos valores medidos

\begin{tabular}{ccc}
\hline $\begin{array}{c}\text { Índice para avaliação do } \\
\text { desempenho do modelo }\end{array}$ & $\begin{array}{c}\text { Classificação da precisão dos dados de vazão e } \\
\text { PBIAS }\end{array}$ \\
NSE & $\%$ & Muito boa \\
Sedimentos, simulados em relação aos valores medidos
\end{tabular}

Fonte: Adaptado de Moriasi et al. (2007). 
Quadro 3. Sensibilidade dos parâmetros em relação à simulação do escoamento

\begin{tabular}{|c|c|c|c|c|}
\hline \multirow{2}{*}{ Parâmetro } & \multirow{2}{*}{ Descrição } & \multirow{2}{*}{ Unidade } & \multicolumn{2}{|c|}{ Valor do parâmetro } \\
\hline & & & PA $(\text { ordem })^{(*)}$ & CJ (ordem) \\
\hline Alpha_Bf & Fator de recessão do escoamento de base & Dia & $0,00(1)$ & $0,00(1)$ \\
\hline Canmx & Armazenamento máximo de água no dossel vegetativo & $\mathrm{mm}$ & $98,17(12)$ & $93,28(10)$ \\
\hline Ch_K2 & Condutividade hidráulica efetiva no canal & $\mathrm{mm} \mathrm{h}^{-1}$ & $164,34(8)$ & $345,30(8)$ \\
\hline $\mathrm{Ch} \_\mathrm{N} 2$ & Coeficiente de rugosidade de Manning (n) do canal & - & $0,04(6)$ & $0,03(2)$ \\
\hline \multirow[t]{3}{*}{$\mathrm{Cn} 2$} & Valor da curva número $(\mathrm{CN})$ do método do Soil & & & \\
\hline & Conservation Service (SCS) - relacionado diretamente & & & \\
\hline & ao grau de antropização da região & - & $35,00(7)$ & $45,61(7)$ \\
\hline Esco & Fator de compensação da evaporação do solo & - & $0,10(3)$ & $0,55(4)$ \\
\hline Gw_Delay & Tempo de retardo do escoamento subterrâneo & Dia & $414,24(5)$ & $126,39(6)$ \\
\hline Gw_Revap & Coeficiente de reevaporação da água subterrânea & & $0,07(10)$ & $0,34(15)$ \\
\hline Gwqmn & $\begin{array}{l}\text { Profundidade limite da água no aquífero raso para } \\
\text { ocorrer fluxo de retorno }\end{array}$ & $\mathrm{mm}$ & $5000,00(4)$ & $231,13(5)$ \\
\hline Revapmn & $\begin{array}{l}\text { Profundidade da água no aquífero raso para ocorrer } \\
\text { percolação }\end{array}$ & $\mathrm{mm}$ & $359,07(11)$ & $246,17(14)$ \\
\hline Sol_Z & Profundidade do solo & $\mathrm{mm}$ & $25,00(2)$ & $1,69(3)$ \\
\hline Surlag & Tempo de retardo do escoamento superficial & Dia & $12,79(9)$ & $10,02(9)$ \\
\hline Usle_C & $\begin{array}{l}\text { Valor do fator C da USLE aplicável ao tipo de cobertura } \\
\text { do solo (valor não calibrado) }\end{array}$ & - & $(* *)$ & $(* *)$ \\
\hline Usle_K & Fator de erodibilidade do solo da USLE & $\mathrm{th} \mathrm{MJ} J^{-1} \mathrm{~mm}^{-1}$ & $(* * *)$ & $(* * *)$ \\
\hline Usle_P & Fator de práticas conservacionistas da USLE & - & 1 & 1 \\
\hline
\end{tabular}

${ }^{(*)}$ Os valores entre parênteses indicam a ordem de importância na análise de sensibilidade para o modelo; ${ }^{(* *)}$ Mata secundária, USLE_C $=0,0010$; Floresta_C=0,0010; Pastagem_C=0,0080; e Cultivo (cana-de-açucar), C=0,3066. (Fonte: Weill \& Sparovek, 2008); ${ }^{(* *)}$ Argissolos Amarelos, USLE_K=0,029; Argissolos Vermelho-Amarelos, USLE_K=0,021; Neossolos Fluvicos, USLE_K=0,025; e Neossolos Quartzarênicos, USLE_K=0,017. Gerados neste estudo.

primeiros relacionados ao escoamento superficial e os restantes à água subterrânea. Essas variações sugerem diferenças entre os processos de geração do escoamento superficial nas duas bacias e uma primeira indicação de que os parâmetros calibrados em uma bacia podem não fornecer bons resultados, quando utilizados em outra.

O uso do solo influencia sobremaneira o valor dos parâmetros calibrados para o escoamento superficial. Deve-se lembrar que existe predominância de vegetação nativa ou regenerada na área de contribuição de PA, quando comparada com a área do trecho desde o médio curso ao baixo curso do Rio Japaratuba Mirim (Figura 2), onde pastagem e cultivo de cana-de-açúcar se sobressaem. Essa vegetação nativa ou regenerada contribui para maior infiltração e, por consequência, menor escoamento superficial, refletindo no valor do parâmetro $C n 2$, pois, apesar de próximos, o valor para a estação PA foi menor do que para a estação CJ, refletindo maior permeabilidade da região de PA. No entanto, a influência de uma área um pouco mais antropizada, que sofreu mecanização e, consequentemente, mais compactada, incrementou o escoamento superficial, em relação à região de contribuição de $\mathrm{PA}$, elevando o valor global de Cn2 para a área de contribuição de CJ.

De acordo com Fontes (1997), os solos nessa bacia possuem textura predominantemente média a argilosa, de baixa condutividade hidráulica, o que resulta na baixa infiltração da água, levando a maior escoamento. Contudo, a presença da vegetação nativa/ renascida na área de contribuição de PA e até mesmo as culturas existentes na região de contribuição de CJ exercem papel fundamental na redução do escoamento superficial e no impacto das gotas de chuva, prevenindo o solo do processo erosivo e incrementando a infiltração. Além disso, o relevo em toda a área da bacia é relativamente plano (Aragão et al., 2011), incentivando o processo de infiltração, sendo outro fator que limita o processo erosivo nessa bacia.

A existência das diferentes formações geológicas na área de contribuição da estação PA (Figura 2c) sugere que, enquanto a porção da Formação Barreiras (litotipo arenito/argilito) serve como área de recarga, a Formação Macururé (litotipo mármore/quartizito) não permite um considerável aporte da água subterrânea para esse trecho do Rio, o que pode ser um dos motivos para a diferença no valor de GWrevap, GWqmm e Revap entre as duas bacias, visto que os valores dos parâmetros GWqmm e Revap para PA foram bem mais altos que para CJ. De acordo com Neitsch et al. (2005), um valor alto para GWqmn implica baixo aporte de água subterrânea para o trecho de rio; ou seja, como esses parâmetros têm grande afinidade entre si, seus valores indicam que existe 
pouca contribuição de água subterrânea para esse trecho do rio (Figura 2c), o que é compatível com as condições geológicas e a região climática da nascente desse rio, região de transição entre agreste e semiárido (Figura 1). O valor de Esco também reflete um pouco da influência da região climática de contribuição de $\mathrm{PA}$, visto que valores próximos a zero sugerem mais evaporação da água no solo nas camadas mais profundas, fato que ocorre em razão da alta insolação naquela região da bacia.

Observou-se que a rotina de autocalibração do modelo não forneceu valores finais adequados para alguns parâmetros, por exemplo, o valor de $C h \_K 2 \mathrm{e}$ o de Sol_Z (Quadro 3). O uso do valor de Ch_K2 levou o modelo a zerar os valores de vazão simulada em diversos cursos de água das bacias. Assim, ao invés de utilizar esse valor, foi usado o valor "padrão" que é zero. Tal fato pode também estar associado ao número de interações considerado no processo (1.000), sendo possível que um número superior dessas, levasse a valores diferentes de $C h \_K 2$, como também dos outros parâmetros. Entretanto, o valor de $S o l \_Z$ foi bem diferente daqueles obtidos por Fontes (1997) e foram desconsiderados nos resultados apresentados a seguir. Dessa forma, os valores calibrados levaram ao resultado apresentado na figura 3, em que se observou que o modelo conseguiu reproduzir bem tanto a forma do hidrograma quanto os picos, fato esse comprovado pelos valores de NSE $(0,83)$ e PBIAS $(8,50 \%)$, o que leva a considerar a calibração como muito boa, fazendo-se uma ressalva aos períodos de estiagem, em que o modelo subestima os valores simulados. Isto pode ser causado pelo fato de $o$ modelo não conseguir reproduzir bem o processo de água subterrânea nesse período, já que essa componente é significativa no período de estiagem.

Em se tratando de validação, feita com dados do período 1989 a 1990, o modelo subestimou os valores de vazão de pico (Figura $3 b$ ) para os maiores eventos; porém, acompanhou a forma do hidrograma e, assim, com índices NSE $(0,66)$ e PBIAS $(38,74 \%)$, a simulação pode ser considerada boa.
A parametrização do modelo SWAT levou em conta as diferenças físicas entre as duas bacias deste estudo e considerou as diversidades de tipo, declividades e usos do solo. Este fato foi bem evidenciado pela simulação das vazões para seção PA, utilizando os parâmetros calibrados para a seção CJ (Figura 4) e pelos índices obtidos $(\mathrm{NSE}=0,41 ; \mathrm{PBIAS}=41,68 \%)$. Uma das explicações para o modelo superestimar os valores simulados (Figuras 4) e o consequente baixo valor de $\mathrm{NSE}=0,41$, deve-se, certamente, à diferença no valor de alguns parâmetros influentes, como é o caso do $C n 2$, visto que, para a estação CJ, o valor foi 45,6 e, para a estação PA, 35. Esse valor de $\mathrm{Cn} 2$ reflete o tipo de solos e o uso do solo para CJ, de forma que, quando $\mathrm{Cn} 2$ foi transferido para PA, provocou escoamento mais alto do que o observado para essa estação.

Apesar de os valores das vazões máximas simuladas não estarem muito próximos aos observados (Figura 4), o modelo conseguiu reproduzir, com fidelidade, o padrão do hidrograma, de modo que os resultados podem ser considerados uma primeira estimativa de vazão, caso não existam dados observados no local.

Com o intuito de verificar se o processo inverso poderia levar a resultados melhores, os parâmetros calibrados para a estação PA foram empregados na bacia de contribuição da estação CJ (Figura 5). O modelo, como esperado, subestimou as vazões máximas e alguns valores de vazão mínima. Atribuise esse comportamento, assim como no caso anterior, à influência, principalmente, do parâmetro associado ao uso e à cobertura do solo (Cn2). Apesar desse comportamento, os índices NSE $(0,66)$ e PBIAS $(38,74 \%)$, considerados bons (Moriasi et al., 2007), refletem os hidrogramas apresentados. Observou-se novamente que, para o período de 1986 a 1988 (utilizado na calibração), o modelo simulou as vazões abaixo dos valores observados para a estação CJ e, para os outros períodos, a vazão simulada foi menor do que a observada, porém acompanhou a variação da vazão observada.
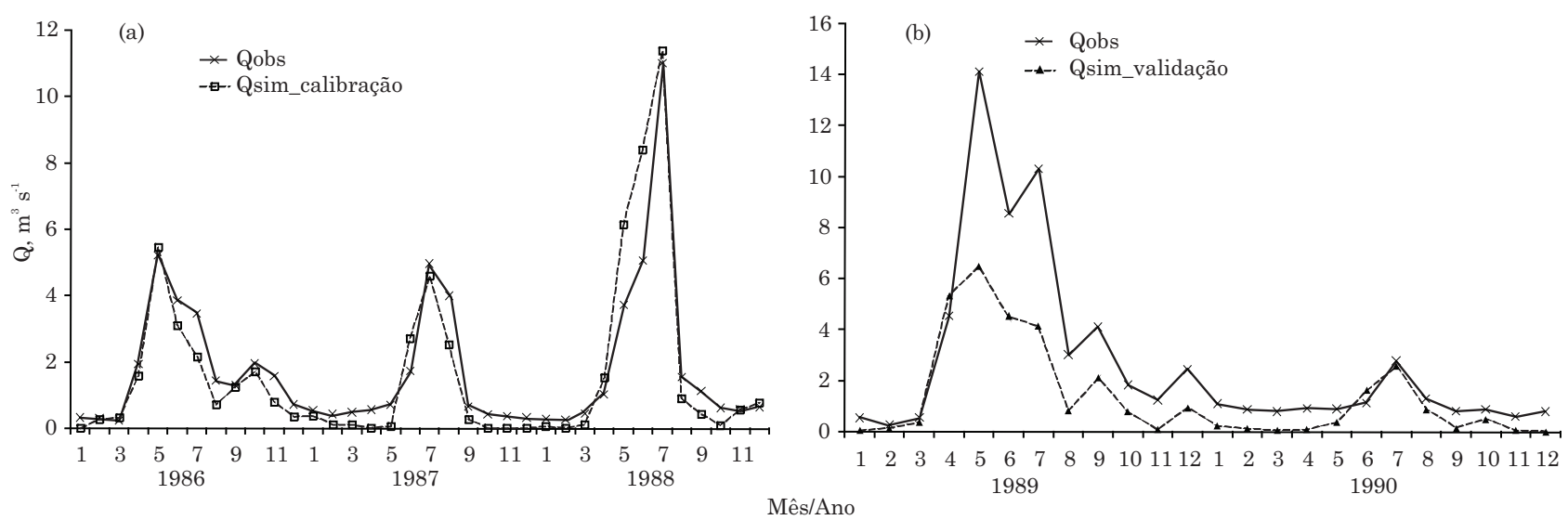

Figura 3. Hidrogramas observados (Qobs) e simulados (Qsim) para calibração (a) e validação (b), com dados do posto Fazenda Cajueiro (1986 a 1990). 


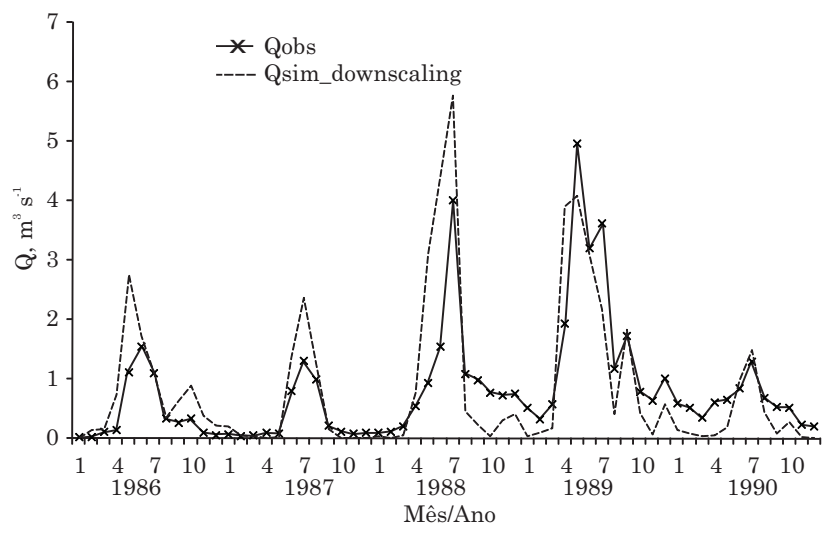

Figura 4. Hidrogramas observados (Qobs) e simulados para o posto Fazenda Pão de Açúcar, com parâmetros calibrados (Qsim_downscaling) na Fazenda Cajueiro (1986 a 1990).

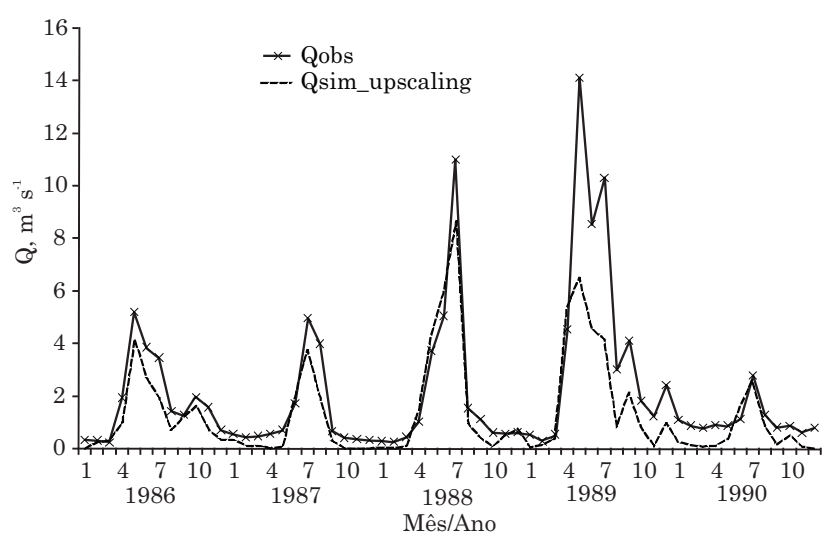

Figura 5. Hidrogramas observados (Qobs) e simulados para o posto Fazenda Cajueiro, com parâmetros calibrados (Qsim_upscaling) na Fazenda Pão de Açúcar (1986 a 1990).

Em termos gerais, a calibração e a validação para vazão simulada para as estações CJ e PA foram satisfatórias, com valores de NSE e PBIAS considerados bons, esperando-se que a simulação da produção de sedimento possa fornecer bons resultados. É interessante notar que apesar das duas bacias estarem aninhadas e na mesma região climática, as transferências dos parâmetros de CJ para PA e de PA para CJ causaram diferença entre os valores observados e calculados, em razão das diferenças no uso do solo, bem como do tipo de solo entre as bacias de contribuição para as estações PA e CJ.

Uma vez que o modelo calibrado produziu resultados satisfatórios das vazões no período de validação (Figura $3 \mathrm{~b}$ ), procedeu-se à simulação hidrossedimentológica, para as duas estações fluviométricas e para o período de dados de vazão de 1986-2000. Por não existirem dados observados da produção de sedimentos, a análise da simulação da erosão teve de ser qualitativa, sendo de interesse principal verificar se a produção de sedimento simulada teve valores compatíveis com as áreas das bacias, conforme tendências descritas na literatura (Lane et al., 1997; Birkinshaw \& Bathurst, 2006; Figueiredo, 2008). Os resultados das simulações das vazões $\left(\mathrm{m}^{3} \mathrm{~s}^{-1}\right)$ e das produções de sedimentos $\left(\mathrm{t} \mathrm{km}^{-2}\right.$ $a^{-1}$ ) anuais estão apresentados no quadro 4 .

Considerando a tendência das simulações, a compatibilidade dos dados de vazão com o uso do solo e a escala da bacia (Lane et al., 1997; Dedkov, 2004; Birkinshaw \& Bathurst, 2006; Figueiredo, 2008), existe proporcionalidade entre os dados de vazão para as duas seções, uma vez que a razão das áreas (CJ = $277,8 \mathrm{~km}^{2} ; \mathrm{PA}=137,3 \mathrm{~km}^{2}$ ), próxima de 2 , foi seguida pela razão entre os valores de vazão média anual nas estações, ou seja, o modelo foi consistente com a variação na escala.

Essa mesma consistência foi seguida pelo modelo de erosão (razão média próxima de 1,5), com exceção do ano de 1998, que apresenta uma razão $\mathrm{SY}_{\mathrm{CJ}} / \mathrm{SY}_{\mathrm{PA}}$ inferior a 1 , significando que na seção jusante a PA a deposição de sedimentos erodidos foi maior que toda a erosão e, consequentemente, a quantidade de sedimentos que chegou a seção CJ foi menor do que a que saiu da seção PA.

Em se tratando de produção de sedimentos, a literatura relata (Lane et al., 1997; Dedkov, 2004; Birkinshaw \& Bathurst, 2006) tendência de diminuição dessa variável com o aumento da área da bacia. Porém, neste estudo, foi visto um efeito inverso (Quadro 4). Tal fato pode ser resultado de diversos fatores associados, como predominância de classes de solos mais suscetíveis à erosão ou usos da terra que promovam menor proteção ao solo (Lane et al., 1997; Birkinshaw \& Bathurst, 2006).

A classe de solo teve papel fundamental na diferença unitária da produção de sedimentos em ambas as bacias, uma vez que na bacia da seção PA predominam os Argissolos Vermelho-Amarelos, cujo coeficiente de erodibilidade (fator $\mathrm{K}$ ) apresenta valores inferiores aos Argissolos-Amarelos, que predominam na porção a jusante da estação Pão de Açúcar (Figura 2b). Associado a isso, acrescenta-se ainda o fato que há crescimento gradativo dos coeficientes de erosividade (fator R) de montante para jusante na estação CJ (Figura 2e), em decorrência dos maiores volumes de precipitação no sentido agreste-litoral da bacia.

É importante citar que as áreas de florestas nativas ou regeneradas contribuem para a redução da desagregação do solo por impacto de gota de chuva, como também pelo arrasto do escoamento superficial, reduzindo assim a produção de sedimento nessa área, o que reflete nos valores baixos para o fator de uso e cobertura do solo (fator C), para as classes Florestas e Matas secundárias (Figura 2f), que ocupam grandes parcelas a montante da estação PA; papel semelhante, porém em menor proporção, exerce a pastagem (Bertoni \& Lombardi Neto, 2005). Contudo, na transição de períodos secos para os chuvosos, o pasto está bastante seco e nem sempre protege o solo a contento. 
Quadro 4. Vazão média anual (Q) e produção de sedimento (SY) para as seções Fazenda Cajueiro (CJ) e Fazenda Pão de Açúcar (PA)

\begin{tabular}{|c|c|c|c|c|c|c|}
\hline \multirow{3}{*}{ Ano } & \multicolumn{4}{|c|}{ Fazenda } & \multirow{2}{*}{\multicolumn{2}{|c|}{ Razão }} \\
\hline & \multirow{2}{*}{$\begin{array}{c}\text { Cajueiro } \\
\mathbf{Q}_{\mathrm{CJ}}\end{array}$} & \multirow{2}{*}{$\begin{array}{c}\text { Pão de Açúcar } \\
\mathbf{Q}_{\mathbf{P A}}\end{array}$} & \multirow{2}{*}{$\begin{array}{c}\text { Cajueiro } \\
\text { SY }_{\text {CJ }}\end{array}$} & \multirow{2}{*}{$\begin{array}{c}\text { Pão de Açúcar } \\
\text { SY }_{\mathrm{PA}} \\
\end{array}$} & & \\
\hline & & & & & $\mathbf{Q}_{\mathrm{CJ}} / \mathbf{Q}_{\mathbf{P A}}$ & $\mathbf{S Y}_{\mathbf{C J}} / \mathbf{S Y}_{\mathbf{P A}}$ \\
\hline & & $\mathrm{s}^{-1}$ & $-\mathrm{t} \mathrm{k}$ & $\mathrm{ano}^{-1}$ & & \\
\hline 1986 & 1,47 & 0,75 & 5,62 & 3,49 & 1,96 & 1,61 \\
\hline 1987 & 0,89 & 0,45 & 4,50 & 2,87 & 1,99 & 1,57 \\
\hline 1988 & 2,52 & 1,29 & 19,09 & 10,62 & 1,96 & 1,80 \\
\hline 1989 & 2,71 & 1,39 & 15,44 & 10,09 & 1,95 & 1,53 \\
\hline 1990 & 0,60 & 0,31 & 0,65 & 0,57 & 1,95 & 1,14 \\
\hline 1991 & 0,90 & 0,46 & 1,75 & 1,27 & 1,95 & 1,37 \\
\hline 1992 & 0,96 & 0,49 & 3,12 & 1,40 & 1,94 & 2,22 \\
\hline 1993 & 0,48 & 0,24 & 0,35 & 0,21 & 1,96 & 1,69 \\
\hline 1994 & 0,84 & 0,43 & 4,10 & 2,44 & 1,97 & 1,68 \\
\hline 1995 & 0,54 & 0,28 & 0,00 & 0,00 & 1,96 & - \\
\hline 1996 & 1,21 & 0,62 & 3,75 & 2,62 & 1,95 & 1,43 \\
\hline 1997 & 0,73 & 0,37 & 2,90 & 1,57 & 2,00 & 1,85 \\
\hline 1998 & 0,46 & 0,24 & 0,53 & 0,60 & 1,91 & 0,89 \\
\hline 1999 & 1,02 & 0,52 & 4,37 & 2,25 & 1,94 & 1,94 \\
\hline 2000 & 1,30 & 0,67 & 6,96 & 3,58 & 1,94 & 1,95 \\
\hline
\end{tabular}

Ainda considerando o uso e a cobertura do solo, a jusante da estação PA, percebe-se o predomínio da ocupação do solo com culturas agrícolas, principalmente cana-de-açúcar. No período de entressafra das usinas de açúcar e, ou, álcool, que acontece de maio a agosto, o solo fica descoberto devido à colheita o que favorece maior movimentação de terra, facilitando a desagregação das partículas de solo e o transporte de sedimentos pelo escoamento, resultando, por sua vez, no incremento dos valores da produção de sedimentos na estação CJ. Tal contribuição está claramente representada no valor mais alto para o fator C (uso e cobertura do solo), se comparado aos valores atribuídos para matas secundárias e florestas. Esse comportamento também foi observado por Lane et al. (1997), Dedkov (2004), Birkinshaw \& Bathurst (2006) e Figueiredo (2008).

\section{CONCLUSÕES}

1. O modelo SWAT foi sensível ao parâmetro Curva Número $(\mathrm{Cn} 2)$ e aos parâmetros relacionados à água subterrânea, denotando a influência do uso e da cobertura do solo e das condições de recarga do aquífero no escoamento superficial.

2. O modelo conseguiu simular satisfatoriamente os hidrogramas observados, uma vez que os parâmetros foram calibrados.

3. Os valores obtidos para os índices de Eficiência de Nash-Sutcliffe(NSE) e de Tendência Percentual(PBIAS) foram considerados satisfatórios para o primeiro caso e insatisfatório para o segundo, nas simulações com os parâmetros transpostos da bacia de contribuição menor (PA) para a bacia maior (CJ) e vice-versa.

4. O modelo conseguiu reproduzir consistentemente a vazão e a produção de sedimentos, com razões aproximadamente constantes, para as condições de uso e cobertura dos solos existentes nas bacias aninhadas.

5. A tendência de diminuição da produção de sedimento com a área da bacia sugerida na literatura não foi verificada.

\section{AGRADECIMENTOS}

Ao CNPq, à FINEP, à Embrapa e à FAPITEC/SE, pelo apoio financeiro; e à ANA e SEMARH/SE, pelos dados fornecidos.

\section{LITERATURA CITADA}

AGÊNCIA NACIONAL DAS ÁGUAS - ANA. Hidro Web: Sistemas de informações hidrológicas. Disponível em: $<$ http://hidroweb.ana.gov.br>. Acesso em: 10 mar. 2012.

ARAGÃO, R.; ALMEIDA, J.A.P.; FIGUEIREDO, E.E. \& SRINIVASAN, V.S. Mapeamento do potencial de erosão laminar na bacia do rio Japaratuba - SE, via SIG. R. Bras. Eng. Agr. Amb., 15:731-740, 2011. 
ARAÚJO, J.C. \& KNIGHT, D.W. A review of the measurement of sediment yield in different scales. R. Esc. Minas, 53:257$265,2005$.

ARAÚJO FILHO, J.C.; LOPES, O.F.; OLIVEIRA NETO, M.B., NOGUEIRA, L.R.Q. \& BARRETO, A.C. Levantamento de reconhecimento de média intensidade dos solos da região dos tabuleiros costeiros e baixada litorânea no Estado de Sergipe. Rio de Janeiro, Embrapa Solos/ Aracaju, Embrapa Tabuleiros Costeiros, 1999. CD-ROM. (Embrapa Solos. Boletim de Pesquisa, 4)

ARNOLD, J.G.; WILLIAMS, J.R. \& MAIDMENT, D.R. Continuous-time water and sediment-routing model for large basins. J. Hydrol. Eng., 121:171-183, 1995.

BALTOKOSKI, V.; TAVARES, M.H.F.; MACHADO, R.E. \& OLIVEIRA, M.P. Calibração de modelo para a simulação de vazão e de fósforo total nas sub-bacias dos rios Conrado e Pinheiro - Pato Branco (PR). R. Bras. Ci. Solo, 34:253$261,2010$.

BALDISSERA, G.C. Aplicabilidade do modelo de simulação hidrológica SWAT (Soil and Water Assessment Tool), para a bacia hidrográfica do Rio Cuiabá/MT. Cuiabá, Universidade Federal do Mato Grosso, 2005. 148p. (Dissertação de Mestrado)

BERTOL, I.; COGO, N.P.; SCHICK, J.; GUDAGNIN, J.C. \& AMARAL, A.J. Aspectos financeiros relacionados às perdas de nutrientes por erosão hídrica em diferentes sistemas de manejo do solo. R. Bras. Ci. Solo, 31:133-142, 2007.

BERTONI, J. \& LOMBARDI NETO, F. Conservação do solo. 5.ed. São Paulo, Ícone, 2005. 355p.

BEVEN, K. Rainfall-Runoff Modelling - The primer. West Sussex, John Wiley \& Sons, 2001. 360p.

BIRKINSHAW, S.J. \& BATHURST, J.C. Model study of the relationship between sediment yield and river basin area. Earth Surf. Process. Landf., 31:750-761, 2006.

DEDKOV, A. The relationship between sediment yield and drainage basin area. In: GOLOSOV, V.; BELYAEV, V. \& WALLING, D.E., eds. Sediment transfer through the fluvial systems, Wallingford, 2004. p.197-204. (IAHS Publ., 288)

DUAN, Q.; SOROOSHIAN, S. \& GUPTA, V. Optimal use of the SCE - UA global optimization method for calibrating watershed models. J. Hydrol., 158:265-284, 1994.

EMPRESA BRASILEIRA DE PESQUISA AGROPECUÁRIA EMBRAPA. Centro Nacional de Pesquisa de Solos. Sistema brasileiro de classificação de solos. 2.ed. Rio de Janeiro, 2006. 306p.

FIGUEIREDO, E.E. Sediment yield modelling at micro-basin and basin scales in semi-arid regions of Brazil. In: SCHMIDT, J.; COCHRANE, T.; PHILLIPS, C.; ELLIOTT, S.; DAVIES, T. \& BASHER, L., eds. Sediment dynamics in changing environments. Wallingford, 2008. p.157-166. (IAHS Publ., 325)

FONTES, A.L. Caracterização da bacia do Rio Japaratuba (SE). Rio Claro, Universidade Estadual Paulista, 1997. 200p. (Tese de Doutorado)
GARBOSSA, L.H.P.; VASCONCELOS, L.R.C.; LAPA, K.R.; BRAINSKI, E. \& PINHEIRO, A. The use and results of the soil and water assessment tool in Brazil: A review from 1999 until 2010. In: INTERNATIONAL SWAT CONFERENCE \& WORKSHOPS, 2011, Toledo. Proceedings... Toledo, Texas A\&M University, 2011. p.127. Disponível em: <http://swat.tamu.edu/conferences/ 2011/>. Acesso em: 10 mar. 2012.

JAYAKRISHNAN, R.; SRINIVASAN, R.; SANTHI, C. \& ARNOLD, J.G. Advances in the application of the SWAT model for water resources management. Hydrol. Proces., 19:749-762, 2005 .

LELIS, T.A.; CALIJURI, M.L. \& FONSECA, A. Análise de sensibilidade e calibração do modelo SWAT aplicado em bacia hidrográfica da Região Sudeste do Brasil. R. Bras. Ci. Solo, 36:623-634, 2012

LANE, L.J.; HERNANDEZ, M. \& NICHOLS, M. Processes controlling sediment yield from watersheds as functions of spatial scale. Environ. Model. Softw., 4:355-369,1997.

LIMA, J.E.F.W.; LOPES, W.T.A.; CARVALO, N.O. \& VIEIRA, M.R. Fluxo de sedimentos em suspensão no exutório de grandes bacias hidrográficas em território brasileiro. In: ENCONTRO NACIONAL DE ENGENHARIA DE SEDIMENTOS, 8., Campo Grande, 2008. Anais... Campo Grande, ABRH, 2008. CD-ROM

MEIRA NETO, A.; FONTES, A.S. \& MEDEIROS, Y.D.P. Aplicabilidade do Modelo SWAT a um Sistema Hidrológico Complexo de Clima Semiárido. In: SIMPÓSIO BRASILEIRO DE RECURSOS HÍDRICOS, 19., Maceió, 2011. Anais... Maceió, ABRH, 2011. CD-ROM

MELO, H.A. Integração entre modelos hidrológicos e sistemas de informações geográficas: Uma avaliação metodológica. Campina Grande, Universidade Federal de Campina Grande, 2010. 200p. (Dissertação de Mestrado)

MERRITT, W.S.; LETCHER, R.A. \& JAKEMAN, A.J. A review of erosion and sediment transport models. Environ. Model. Softw, 18:761-799, 2003.

MIRANDA, E.E. Brasil em Relevo. Campinas, Embrapa Monitoramento por Satélite, 2005. Disponível em: <http:// www.relevobr.cnpm.embrapa.br>. Acesso em: 5 Jun. 2009.

MORIASI, D.N.; ARNOLD, J.G.; van LIEW, M.W.; BINGNER, R.L.; HARMEL, R.D. \& VEITH, T.L. Model evaluation guidelines for systematic quantification of accuracy in watershed simulations. Trans. ASABE, 50:885-900, 2007.

NEITSCH, S.L.; ARNOLD, J.G.; KINIRY, J.R. \& WILLIAMS, J.R. Soil and water assessment tool: Theoretical documentation - version 2005. Grassland, Soil and Water Research Laboratory - Agricultural Research Service; Blackland Research Center - Texas Agricultural Experiment Station, 2005. 494p.

PUGLIESI, A.C.V.; MARINHO, M.A.; MARQUES, J.F. \& LUCARELLI, J.R.F. Valoração econômica do efeito da erosão em sistemas de manejo do solo empregando o método custo de reposição. Bragantia, 70:113-121, 2011.

SECRETARIA DE ESTADO DO MEIO AMBIENTE E DOS RECURSOS HÍDRICOS - SEMARH. Atlas digital de recursos hídricos. Aracaju, SEMARH/SRH, 2004. CD-ROM 
SINGH, V.P. \& WOOLHISER, D.A. Mathematical modeling of watershed hydrology. J. Hydrol. Eng., 7:520-530, 2002.

STRAUCH, M.; BERNHOFER, C.; KOIDE, S.; VOLK, M.; LORZ, C. \& MAKESCHIN, F. Using precipitation data ensemble for uncertainty analysis in SWAT stream flow simulation. J. Hydrol., 414-415:413-424, 2012.

van GRIENSVEN, A.; MEIXNER, T.; GRUNWALD, S.; BISHOP, T.; DILUZIO, M. \& SRINIVASAN, R. A global sensitivity analysis tool for the parameters of multivariable catchment models. J. Hydrol., 324:10-23, 2006.

WALLING, D.E. \& WEBB, B.W. Erosion and sediment yield: A global overview. In: WALLING, D.E. \& WEBB, B.W., eds. Erosion and sediment yield: Global and regional perspective. Wallingford, 1996. p.3-19. (IAHS Publ., 236)
WEILL, M.A.M. \& SPAROVEK, G. Erosion study in the ceveiro watershed (Piracicaba, SP): I - Estimation of soil loss rates and sensitivity factor analysis of the USLE model. R. Bras. Ci. Solo, 32:801-814, 2008.

WILLIAMS, J.R. Sediment routing for agricultural watersheds. J. Am. Water Res. Assoc., 11:965-974, 1975.

WISCHMEIER, W.H.; JOHNSON, C.B. \& CROSS, B.V. A soil erodibility nomograph for farmland and construction sites. J. Soil Water Conserv., 26:189-193, 1971.

WINCHELL, M.; SRINIVASAN, R.; DI LUZIO, M. \& ARNOLD, J. ARCSWAT 2.3.4 interface for SWAT2005: User's guide. Grassland, Soil and Water Research Laboratory/ Agricultural Research Service/Blackland Research Center - Texas/Agricultural Experiment Station, 2009. 494p. 